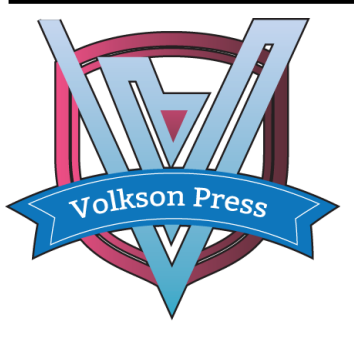

Contents List available at VOLKSON PRESS

New Materials and Intelligent Manufacturing (NMIM)

DOI : http://doi.org/10.26480/icnmim.01.2018.121.123

Journal Homepage: https://topicsonchemeng.org.my/

ISBN: 978-1-948012-12-6

\title{
QUALITY CONTROL OF SAN'AO TABLETS VIA X-RAY DIFFRACTION AND ELEC TRON SPIN RESONANCE TECHNIQUES
}

\author{
Yezhuo Zhao, Dayong Lu* \\ Key Laboratory for Special Functional Materials in Jilin Provincial Universities, Jilin Institute of Chemical Technology Chengde Street 45, \\ Jilin 132022, China. \\ *Corresponding Author Email: dylu@jlict.edu.cn
}

This is an open access article distributed under the Creative Commons Attribution License, which permits unrestricted use, distribution, and reproduction in any medium, provided the original work is properly cited

\section{ARTICLE DETAILS}

\section{Article History:}

Received 26 June 2018 Accepted 2 July 2018

Available online 1 August 2018

\section{ABSTRACT}

Both X-ray diffraction (XRD) and electron spin resonance (ESR) techniques were used to investigate San'ao tablets and their ingredients. Three XRD peaks at $2 \theta=16.10^{\circ}, 22.52^{\circ}$ and $34.46^{\circ}$, which arise mainly from a kind of pharmaceutic excipient-microcrystalline cellulose, are characteristic of San'ao tablets. Calcium oxalate monohydrate and calcium oxalate without crystalliferous water, as two different forms of crystalline metabolites, are identified to exist in ephedra and licorice, respectively. The ESR spectra of San'ao tablets mainly consist of those of four kinds of Chinese herbal ingredients-ephedra, bitter almonds, licorice, and ginger, independent of pharmaceutic excipients. The $\mathrm{Mn}^{2+}$ signal in San'ao tablets mainly arise from ephedra and licorice. The combination of XRD and ESR can provide a new method for quality control of San'ao tablets.

\section{KEYWORDS}

San'ao tablets, X-ray diffraction, electron spin resonance, quality control.

\section{INTRODUCTION}

San'ao tablets have remarkable curative effect on acute bronchitis and cough induced by attacked lungs with cold wind, which have been widely used in clinical treatment [1-3]. It is composed of four kinds of Chinese herbal ingredients - Ephedra, bitter almonds, licorice, and ginger. San'ao tablets have good curative effect on body's lung functions. The symptoms of cough are divided into severe cough, white and thin phlegm, and phlegmatic cough [4]. The initial standard is to have quantitative indicator of ephedrine hydrochloride and to identify the main ingredient of bitter almonds - amygdalin using HPLC [5]. However, there is no standard to identify ginger and licorice to date.

It is well known that the XRD technique can provide the whole structural information about Chinese traditional patent and herbal medicines [610]. In this work, both XRD and ESR techniques are combined to explore a new method for quality control of San'ao tablets.

\section{EXPERIMENTAL}

\subsection{Sample's preparation}

San'ao tablets and their four kinds of Chinese herbal ingredients were provided by Jichuan Pharmaceutical Group Co. Ltd. and Hebei Zhijia Pharmaceutical Co. Ltd., respectively. The detail information of San'ao tablets and their ingredients is listed in Table 1.

Table 1: The information of San'ao tablets and their ingredients.

\begin{tabular}{|l|l|l|l|}
\hline Name & Serial Number & Production Date & Manufacturer \\
\hline San'ao tablets & 1704023 & April 11, 2017 & Jichuan Pharmaceutical Group \\
\hline Ephedra & $1708002 \mathrm{~S}$ & August 6, 2017 & Hebei Zhijia Pharmaceutical \\
\hline Bitter almonds & $1701001 \mathrm{~W}$ & January 3, 2017 & Hebei Zhijia Pharmaceutical \\
\hline Licorice & $1604002 \mathrm{~S}$ & April 16, 2016 & Hebei Zhijia Pharmaceutical \\
\hline Ginger & $1605008 \mathrm{~S}$ & May 16, 2016 & Hebei Zhijia Pharmaceutical \\
\hline
\end{tabular}

San'ao tablets peeled and the above four kinds of Chinese herbal ingredients were pulverized and ground in agate mortar for $30 \mathrm{~min}$. The powders were screened with 100 -mesh screen to prepare fines. These fines were placed in an electronic moisture-proof cabinet for XRD or ESR measurements. The humidity in the cabinet was adjusted to $1 \%$ and the storage temperature of $18^{\circ} \mathrm{C}$.

Microcrystalline cellulose (Huzhou Linghu Xinwang Chemical Co. Ltd., Serial number: 20150305) and sodium carboxymethyl cellulose (Ningbo
Haishu Cellulose Derivatives Factory, Serial number: 20160901) are often used as the pharmaceutical excipients of San'ao tablets.

\subsection{Characterizations}

XRD data was collected at room temperature (RT) between $5^{\circ} \leq 2 \theta \leq 70^{\circ}$ and in steps of $0.02^{\circ}$ for 3 s per step using a DX-2700 X-ray diffractometer (Dandong Haoyuan). Electron spin resonance (ESR) measurements were performed at RT using an EMX Plus X-band spectrometer (Bruker) operating at $9.84 \mathrm{GHz}$. 


\section{RESULTS AND DISCUSSION}

\subsection{XRD of San'ao tablets}

The powder XRD patterns of San'ao tablets, main ingredients, and excipients are shown in Figure 1. San'ao tablets exhibit a broad dispersion peak at $2 \theta=16.10^{\circ}$ and two sharper peaks at $2 \theta=22.52^{\circ}$ and $34.46^{\circ}$. No diffraction peaks exist above $35^{\circ}$. A strong dispersion band centered at $2 \theta$ $=19.80^{\circ}$ appears in bitter almonds. This band can be neglected because no similar shoulder peak exists in the XRD patterns of San'ao tablets. A series of sharp crystalline peaks in ephedra is also absent for San'ao tablets. Thus, these three diffraction peaks at between $2 \theta=15^{\circ}$ and $35^{\circ}$ arise mainly from microcrystalline cellulose.

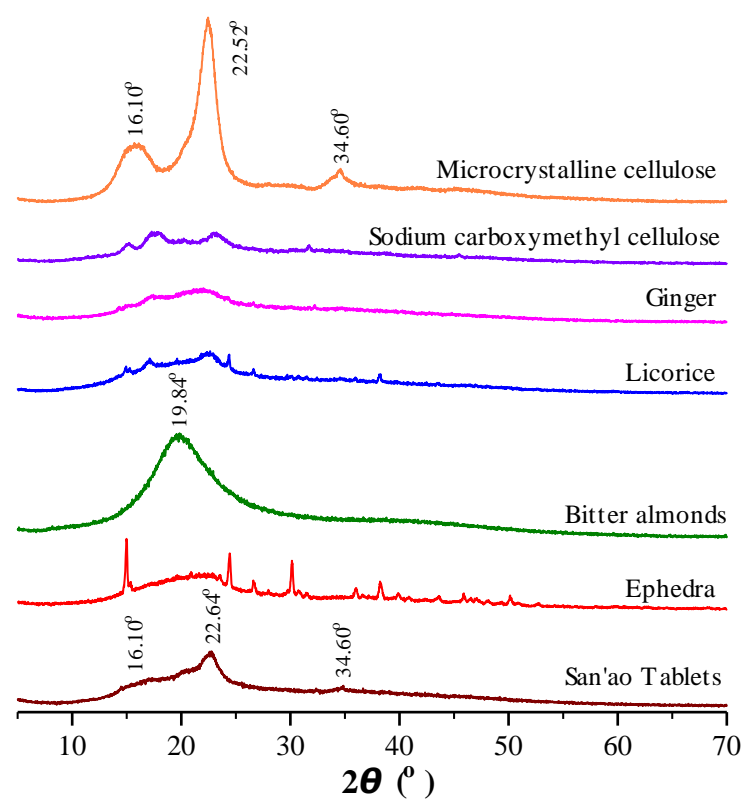

Figure 1: Powder XRD patterns of San'ao tablets, main ingredients, and excipients.

\subsection{Identifications on calcium oxalate in ephedra and licorice using XRD}

It is known from Chinese Pharmacopoeia that the tiny calcium oxalate sandy- and prismatic-crystals, as the plant's own metabolites, exist in the outer walls of ephedra epidermis cells, cortical parenchyma cells, and fibers [7,11-13]. We attempt to identify the series of sharp diffraction peaks in ephedra and licorice according to PDF cards from International Powder Diffraction Center Database. The powder XRD patterns of ephedra and the simulated calcium oxalate monohydrate (PDF 75-1313) are shown in Figure 2. This comparison reveals that the most XRD peaks of ephedra arise from calcium oxalate monohydrate with a monoclinic structure because of matching in main XRD peak positions of (100), (040), (200), (-223), (123), and (300) etc., which is in accordance with Zheng Xiaowei et al's report [7].

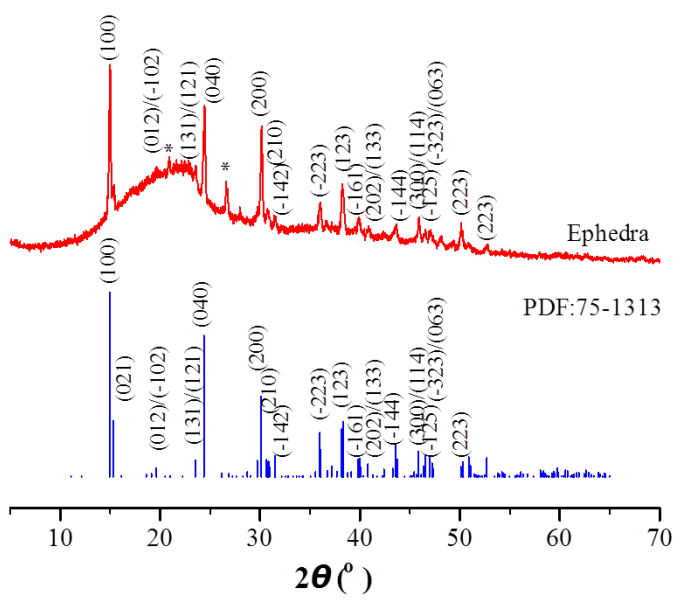

Figure 2: Powder XRD patterns of ephedra and simulated calcium oxalate monohydrate (PDF 75-1313).

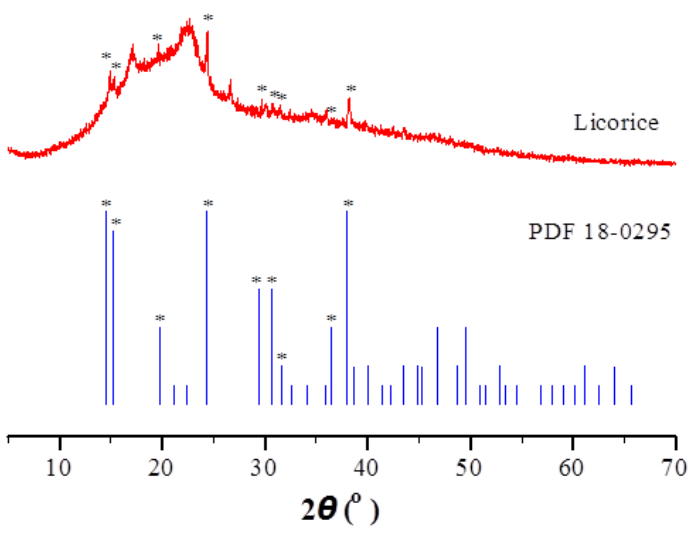

Figure 3: Powder XRD patterns of licorice and simulated calcium oxalate (PDF 18-0295).

Two peaks denoted by asterisk are caused by silica $\left(\mathrm{SiO}_{2}\right)$ with a hexagonal structure (PDF 86-2237), which come from under-cleaning ephedra soil impurities. Although XRD cannot detect the vegetative ingredients in ephedra due to dispersion feature of XRD peak around $2 \theta=23^{\circ}$, the diffractions from monoclinic calcium oxalate monohydrate and hexagonal silica are characteristic of ephedra [6].

Although some peaks of ephedra and licorice overlap, there is no any obvious diffraction peak above $40^{\circ}$ for licorice (Figure 1). This fact illustrates that two series of diffraction peaks in ephedra and licorice arise from different forms of calcium oxalate. It is also known that the parenchyma cells surrounding licorice phloem generally contain calcium oxalate prismatic-crystals [14]. Figure 3 gives the powder XRD patterns of licorice and simulated calcium oxalate (PDF 18-0295). It is obvious that a series of diffraction peaks in licorice arise from calcium oxalate without crystalliferous water, because two sets of diffraction peaks marked by asterisk "*" show astonishing overlap.

No XRD peaks from calcium oxalate monohydrate, silica, and calcium oxalate appear in San'ao tablets (Figure 1), which indicates that these impurities can be removed in the process of manufacture. The XRD feature of San'ao tablets arise from the two main contributions: (1) three main peaks from microcrystalline cellulose and (2) dispersion band around $2 \theta$ $=23^{\circ}$ from four kinds of Chinese herbal ingredients - Ephedra, bitter almonds, licorice, and ginger.

\subsection{ESR identification on San'ao tablets}

The EPR spectra of San'ao tablets and their major ingredients are shown in Figure 4. The $g$ value of isolated free electrons is 2.003 [15]. In Chinese herbal medicines, the resonance absorption of free radicals often occurs at $g=\sim 2.00$ [16]. This signal also appears in each Chinese herbal ingredient of San'ao tablets.

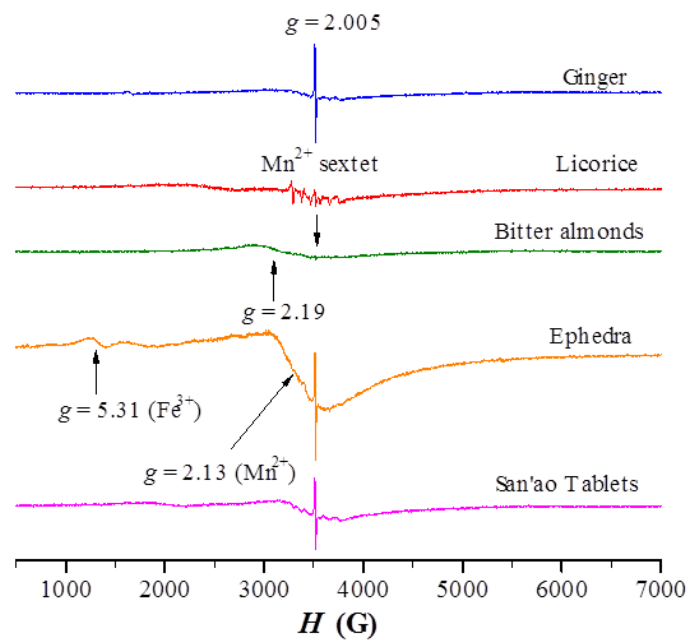

Figure 4: EPR spectra of San'ao tablets and their major ingredients. 
A strongest sextet signal in the vicinity of $H=3500 \mathrm{G}$ for licorice originates from $\mathrm{Mn}^{2+}[17,18]$. It has been reported that ginger and ephedra also contain manganese (Mn) [19-22]. A broad $g=2.13-2.19$ signal between 3100 and $3600 \mathrm{G}$ appears in ephedra and bitter almonds. This signal is attributed to the resonance absorption induced by the higher concentration of $\mathrm{Mn}^{2+}$ as compared to licorice. The $g=2.19$ signal in bitter almonds, which does arise from $\mathrm{Mn}$, is unclear owing to hundreds of organic compositions in this herb. In addition, a $g=5.31$ signal in ephedra is assigned to $\mathrm{Fe}^{3+}$ [23]. No ESR response occurs in microcrystalline cellulose and sodium carboxymethyl cellulose except for a $g=\sim 2.00$ signal. The ESR of San'ao tablets consist of those of four kinds of Chinese herbal ingredients and two kinds of pharmaceutic excipients, irrespective of the resonance absorption of free radicals. On the basis of these investigations, $\mathrm{Mn}^{2+}$ signal in San'ao tablets mainly arise from ephedra and licorice.

\section{CONCLUSION}

Both XRD and ESR techniques were used to investigate San'ao tablets and their ingredients. A broad dispersion peak at $2 \theta=16.10^{\circ}$ and two sharper peaks at $2 \theta=22.52^{\circ}$ and $34.46^{\circ}$ are characteristic of XRD of San'ao tablets, which arise mainly from a kind of pharmaceutic excipientmicrocrystalline cellulose. The metabolites of calcium oxalate in two different crystalline types are identified to exist in ephedra and licorice, corresponding to calcium oxalate monohydrate and calcium oxalate without crystalliferous water, respectively. The ESR spectra of San'ao tablets mainly consist of those of four kinds of Chinese herbal ingredients-ephedra, bitter almonds, licorice, and ginger, independent of pharmaceutic excipients. The $\mathrm{Mn}^{2+}$ signal in San'ao tablets mainly arise from ephedra and licorice. The combination of XRD and ESR can provide a new method for quality control of San'ao tablets.

\section{ACKNOWLEDGMENTS}

This work was funded by the projects of the National Natural Science Foundations of China (21271084) and Changbai Mountain Scholar Distinguished Professor (2015047).

\section{REFERENCE}

[1] Lin, J.L., Cao, L.W. 2017. Clinical observation on treatment of acute bronchitis in children with San'ao tablets combined with western medicine. Maternal and Child Health Care of China, 32 (7), 1496-1498.

[2] Wang, Y.P., Sun, H. 2014. Observation on the efficacy of San'ao tablets in treating acute attack of chronic bronchitis. China Practical Medicines, 9 (16), $160-161$.

[3] Chen, Q., Zhang, W., Zhang, X.C. 2012. Treatment of acute and chronic bronchitis with syndrome of wind-cold attacking lung with San'ao tablets: An analysis of 80 cases. Journal of Clinical Medicine in Practice, 16 (1), 8788.

[4] National Pharmacopoeia Committee. 2015. Pharmacopoeia of People's Republic of China. Part 1 (pp. 133-134). Beijing: China Medical Science and Technology Press.

[5] National Food and Drug Administration. 2009. State Food and Drug Administration Standard, YBZ1081.

[6] Lu, D.Y., Wang, Y., Chang, W. 2017. Study on Shenyang Hongyao tablet using temperature-dependent X-ray diffraction and electron paramagnetic resonance techniques. Lishizhen Medicine and Materia Medica Research, 28 (2), 384-388.

[7] Zheng, X.W., Lv, Y., Zhao, B. 2000. X-ray diffraction Fourier analysis of Niuhuangjiedu tablets. Chinese Journal of Pharmaceutical Analysis, 20 (3), 202-205.

[8] Shi, K.B., Sheng, Z.H. 2007. Identification of ligustrum lucidum Ait. by X-ray diffraction Fourier pattern method. Journal of Chinese Medicinal Materials, 30 (6), 643-645.

[9] Gao, X.H. 2005. Study on Methodology of XRD Fingerprinting of Chinese Herbal Medicine and Establishment of XRD Fingerprint Database of Traditional Chinese Medicine. Master Thesis. Sichuan University.
[10] Feng, Y.H., Yang, S.Y., Wang, S.C., Gong, N.B., Lv, Y. 2011. Analysis on X-ray Diffraction Fourier Fingerprint Pattern of RHIZOMA PINELLIAE. Journal of Anhui Agricultural Sciences, 39 (29), 17874-17876.

[11] Lv, Y., Zheng, Q.T., Wu, N., Wang, G.L. Tian, J.G., Zhang, J., Chen, D.C. 1997. Studies on X-Ray Diffraction Pattern of Traditional Chinese Medicinal Materials. Acta Pharmaceutica Sinica, 32 (3), 193-196.

[12] Gong, N.B., Zheng, X.W., Wang, G.L., Zhang, J., Lv, Y. 2007. Identification on Chinese materia medicacape jasmine by X-ray diffraction fourier fingerprint pattern method. Modern Instruments, 3, 22-24.

[13] National Pharmacopoeia Committee. 2015. Pharmacopoeia of People's Republic of China. Part 1 (pp. 320-321). Beijing: China Medical Science and Technology Press.

[14] National Pharmacopoeia Committee. 2015. Pharmacopoeia of People's Republic of China. Part 1 (pp. 86-87). Beijing: China Medical Science and Technology Press.

[15] Zhao, B.L. 2009. Application of Electron Spin Resonance Technology in Biology and Medicine (p. 3). First edition, HeFei: University of Science and Technology of China Press.

[16] Zoleo, A., Vecchia, F., Brustolon, M. 2008. Characterization of ancient and modern papers by CW-EPR spectroscopy. Applied Magnetic Resonance, 35, 213-223.

[17] Lu, D.Y., Sun, X.Y., Liu, B. Zhang, J.L., Ogata, T. 2014. Structural and dielectric properties, electron paramagnetic resonance, and defect chemistry of Pr-doped $\mathrm{BaTiO}_{3}$ ceramics. Journal of Alloys and Compounds, $615,25-34$

[18] Lu, D.Y., Liu, Q.L., Ogata T. Sun, X.Y., Wang, X. 2011. Tetragonal phase stabilization caused by $\mathrm{Pr}$ ions in $\mathrm{Ba}\left(\mathrm{Ti}_{0.99} \mathrm{Mn}_{0.01}\right) \mathrm{O}_{3}$ with mixed phases. Japanese Journal of Applied Physics, 50 (3), 035806.

[19] Huang, J., Ni, J.B. 1997. Study on trace elements $\mathrm{Zn}, \mathrm{Cu}$, Fe, and $\mathrm{Mn}$ in traditional Cinese medieine. Guangdong Trace Elements Science, 9, 1317.

[20] Yu, J.H., He, R.R., Zhao, J.M., Li, Y.M., Liu, X.M., Jia, F. 2014. Three kinds of treatment of urticaria TCM $\mathrm{Fe}, \mathrm{Cu}, \mathrm{Mn}$ determination of trace elements. Studies of Trace Elements and Health, 31 (2), 32-33.

[21] Liu, C.L., Yin, Y., Zhang, S.H., Li, Q., Chen, M., Guo, X. 2014. Study on correlation between trace elements and active ingredient in Glycyrrhizae Radix Rhizoma. China Journal of Chinese Materia Medica, 39 (17), 3335 3338.

[22] Gao, S.Y., Jia, J., Bai, B.L., Zhang, L.P., Cheng, H. 1998. Study on the content of iron, manganese, zinc and copper in Chinese herbal medicines such as Ginseng. Ginseng Research, 2, 35-36.

[23] Kutty, T.R.N., Murugaraj, P., Gakbhiye, N.S. 1985. EPR evidence for activation of trap centers in PTCR $\mathrm{BaTiO}_{3}$ ceramics. Materials Research Bulletin, 20, 565-574. 